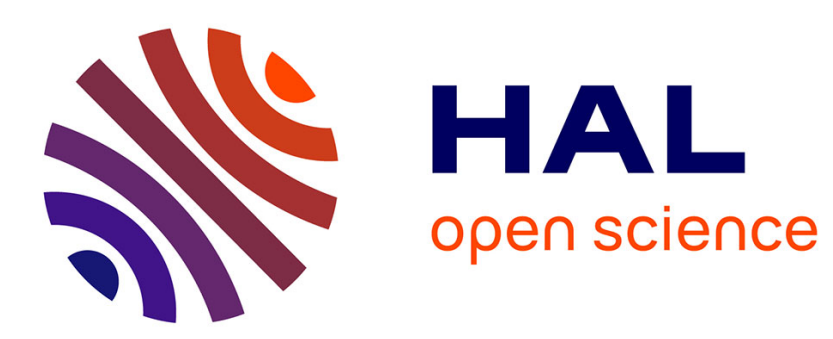

\title{
La notion scientifique de complémentarité et l'objectivité du discours en science et en théologie
}

Jacques Fantino

\section{To cite this version:}

Jacques Fantino. La notion scientifique de complémentarité et l'objectivité du discours en science et en théologie. Revue des Sciences Religieuses, 2003, 77, pp.389-412. 10.3406/rscir.2003.3680 . hal01389788

\section{HAL Id: hal-01389788 \\ https://hal.science/hal-01389788}

Submitted on 8 Nov 2016

HAL is a multi-disciplinary open access archive for the deposit and dissemination of scientific research documents, whether they are published or not. The documents may come from teaching and research institutions in France or abroad, or from public or private research centers.
L'archive ouverte pluridisciplinaire $\mathbf{H A L}$, est destinée au dépôt et à la diffusion de documents scientifiques de niveau recherche, publiés ou non, émanant des établissements d'enseignement et de recherche français ou étrangers, des laboratoires publics ou privés. 


\section{La notion scientifique de complémentarité et l'objectivité du discours en science et en théologie} Jacques Fantino

\section{Résumé}

L'article propose d'analyser l'objectivité du discours en science et en théologie à l'aide de la notion de complémentarité au sens où $\mathrm{N}$. Bohr l'a définie en physique.

\section{Abstract}

In this paper is examined the objectivity of scientific and theological assertions using the notion of complementarity as defined by N. Bohr.

Citer ce document / Cite this document :

Fantino Jacques. La notion scientifique de complémentarité et l'objectivité du discours en science et en théologie. In: Revue des Sciences Religieuses, tome 77, fascicule 3, 2003. pp. 389-412;

doi : 10.3406/rscir.2003.3680

http://www.persee.fr/doc/rscir_0035-2217_2003_num_77_3_3680

Document généré le 03/06/2016 


\section{LA NOTION SCIENTIFIQUE \\ DE COMPLÉMENTARITÉ ET L'OBJECTIVITÉ DU DISCOURS EN SCIENCE ET EN THÉOLOGIE}

La théologie chrétienne parle de Dieu et de son action dans le monde. La référence à Dieu et à son action pose la question de l'objectivité du discours théologique. Comment apprécier celle-ci alors que la réalité visée ne relève pas de l'expérience quotidienne? La théologie n'est pas seule à être dans cette situation. On retrouve une problématique analogue avec le discours scientifique. Le réel qu'étudie la science n'est pas identifiable d'emblée, notamment dans la physique moderne, avec le réel quotidien de l'être humain. Le parallélisme semble pourtant s'arrêter là, car la théologie chrétienne possède encore un autre trait surprenant en tenant des propos qui, vus de l'extérieur, peuvent apparaître contradictoires. C'est le cas, par exemple, des affirmations suivantes : Dieu est un et trine, le Christ Jésus est vrai Dieu et vrai homme, l'accomplissement du dessein de Dieu est déjà effectué et pourtant pas encore achevé. Or, là aussi, une situation semblable existe en physique quantique et l'un de ses fondateurs, Niels Bohr, a inventé la notion de complémentarité pour exprimer et réguler cette condition nouvelle du discours scientifique.

Des théologiens ont repris cette notion de complémentarité au sens où Niels Bohr l'a définie en physique : exprimer une approche radicalement nouvelle de la réalité physique au moyen d'un langage construit en ordonnant ensemble des éléments appartenant à la tradition scientifique, mais auparavant séparés car contradictoires. Appliquée à la théologie, la complémentarité peut lui apporter, selon ces théologiens, d'une part un moyen de se construire à nouveaux frais par rapport à sa propre tradition et par rapport à l'environnement philosophique actuel et d'autre part une possibilité d'insérer le discours théologique dans la culture.

Nous verrons au passage la justesse de cette hypothèse. Mais, ce qui nous retiendra davantage, c'est l'éclairage que la notion de complémentarité apporte à celle d'objectivité appliquée en science ou en théo- 
logie. La première étape de la réflexion consiste à préciser la notion de complémentarité telle que Bohr l'a utilisée. Ensuite, nous verrons l'extension de la notion à d'autres disciplines, principalement à la théologie.

\section{La complémentarité introduite par Bohr}

Il faut commencer par écarter le sens habituel de la complémentaritć selon lequel deux approches complémentaires concernant la même réalité constituent deux points de vue qui s'ajoutent l'un à l'autre ou deux éléments qui peuvent être mis ensemble. Dans cette perspective, science et théologie sont perçus comme tenant sur le monde des propos complémentaires, même si parfois ils apparaissent inconciliables (1). La complémentarité selon Niels Bohr est différente.

Pour la comprendre, il faut revenir aux origines de la mécanique quantique dans les années 1900. Les physiciens, dont Bohr, n'arrivaient pas à décrire correctement le comportement des atomes à partir des concepts et des lois de la physique classique (2). De profonds désaccords apparaissaient en effet entre les résultats expérimentaux concernant les phénomènes atomiques et les prévisions données par les premières tentatives de formulation à partir de la physique classique.

Les observations, pour être interprétées correctement, amenèrent tout d'abord les physiciens à supposer que, contrairement à ce qu'enseigne la physique classique, l'énergie est échangée sous forme de quantités discrètes et indivisibles, appelées quantas. L'interprétation des phénomènes quantiques révéla ensuite qu'un système quantique ne peut pas être appréhender théoriquement à l'aide d'un seul formalisme tiré de la physique classique, mais que, selon les conditions expérimentales, il fallait recourir tantôt à une formulation ondulatoire, tantôt à une formulation corpusculaire. Ainsi, l'interprétation des observations effectuées sur un même système quantique requiert des formulations théoriques contradictoires (3). En effet le concept de corpuscule évoque une entité limitée spatialement, voire à un point, alors que celui d'onde renvoie au contraire à celle d'une réalité étendue dans l'espace, éventuellement infinie.

(1) Science et foi sont deux approches complémentaires dans ce sens dans le livre de G. Theissen, Biblical Faith. An Evolutionary Approach, Philadelphia, Fortress Press, 1985.

(2) Cette expression signifie ici la physique non quantique.

(3) Voir A. MF.ssiah, Mécanique quantique, 2 vol., Paris, Dunod, 1962 et 1964., vol. 1, p. 97. Cet ouvrage demeure une des meilleures présentations en langue française de la physique quantique. 
Pourquoi ces deux aspects, et eux deux seulement? Cela tient d'abord au formalisme développé par la mécanique quantique à partir de celui de la mécanique classique. Selon celle-ci, le mouvement d'une particule et toutes ses propriétés sont entièrement déterminés à partir de la connaissance de sa position dans l'espace à un instant donné et de sa vitesse, ou de son impulsion, à ce même instant. Toutes les autres grandeurs physiques peuvent en effet se déduire de la position et de la vitesse à l'aide des équations de la physique classique. Par conséquent, le formalisme classique repose en dernier ressort sur la position et la vitesse de l'objet considéré, ici l'atome. Retenons que la connaissance du mouvement est complète si l'on connaît la position et la vitesse. Il s'agit de les mesurer.

Or, l'information qu'on peut obtenir sur les systèmes atomiques ou subatomiques proviennent de deux types d'expérience : des collisions ou bien l'absorption ou l'émission d'un rayonnement électromagnétique. Dans le premiers cas de figure, on fait appel à une conceptualisation de ces systèmes sous forme de corpuscules, de «boules de billard » microscopiques. Dans l'autre cas, on recourt au modèle d'un oscillateur capable d'émettre ou de recevoir une onde. Dans un cas le formalisme est construit à partir de la notion de corpuscule, dans l'autre à partir de celle d'onde (4). Ainsi, selon le type d'expérience effectué, le formalisme qui permet de l'interpréter est différent. Telle est la leçon de la physique quantique. La possibilité d'utiliser les concepts de la physique classique est à ce prix. Aucun des deux formalismes, "corpuscule » ou « onde », n'est capable à lui seul de rendre compte de toutes les situations expérimentales, les deux sont nécessaires.

De la dualité des types d'expérience et des formalismes découle par conséquent celle des observations possibles. Ainsi, la mesure de la position relève du formalisme « corpuscule », celle de la vitesse, ou ce qui revient au même, de l'impulsion fait appel au formalisme « onde ». Position et vitesse ne peuvent alors être déterminées en même temps à la différence de ce qui se passe en physique classique où il est possible, au moins en droit, de connaître ces deux grandeurs simultanément. La dualité « onde »- « corpuscule » provient ainsi de ce que l'observation dépend des conditions expérimentales d'une manière complètement différente par rapport à la physique classique à cause de l'indivisibilité du quantum d'action. La discontinuité qui en résulte dans les échanges d'énergie fait qu'on ne peut connaître à la fois la position et la vitesse. On peut mesurer par exemple la vitesse, puis la position. Mais, ce faisant, la perturbation introduite par la mesure de

(4) Voir E. MACKINNON, "Complementarity ", in W. Mark Richardson, Wesley J. Wildman (ed.), Religion and Science. History, Method, Dialogue, New York/London, Routledge, 1996, p. 264. 
la position change d'une manière indéterminable la vitesse au point d'introduire une rupture dans la description causale du comportement dynamique de la particule (5). Au cœur du changement apporté par la physique quantique figure la perturbation due à l'interaction entre le phénomène observé et l'instrumentation et qui ne peut plus être négligée. La finitude du quantum d'action empêche de poser une distinction nette entre un phénomène et l'instrumentation par laquelle il est observé (6). il n'est plus possible en physique quantique de considérer d'un côté l'instrument de mesure et de l'autre le système observé. Cette situation oblige à mettre en place un nouveau mode de description et de compréhension des phénomènes que Bohr appelle complémentarité (7). La complémentarité exprime la nécessaire prise en compte de l'interaction qui se produit entre le système observé et l'instrument lors de toute mesure. Ainsi, en physique quantique, la représentation d'un phénomène n'est plus indépendante des moyens d'observation, entrâ̂nant l'impossibilité de toute séparation nette entre le comportement des objets atomiques et leur interaction avec les instruments de mesure servant à définir les conditions sous lesquelles le phénomène se manifeste (8). Il s'agit là d'une limitation fondamentale (9). Des mesures effectuées sur un même système physique dans des conditions expérimentales différentes renvoient à des conceptualisations qui ne peuvent être rassemblées en une seule présentation synthétique du système comme c'est le cas en physique classique. La complémentarité revient à appliquer alternativement différents concepts classiques qui mèneraient à la contradiction s'ils étaient utilisés simultanément (10).

\section{L'extension de la complémentarité de la physique à la théologie}

Bohr a lui-même proposé d'appliquer la notion de complémentarité à d'autres domaines, comme par exemple la biologie, la psychologie, l'éthique (11). Ces extensions ne sont qu'esquissées par Bohr, mais on peut en dégager le trait principal. Il s'agit à chaque fois de considérer ensemble deux compréhensions incompatibles de la même réalité. En

(5) Voir N. BoHr, Atomic Theory and the Description of Nature, Cambridge, Cambridge University Press, 1934 (réimpression, 1961), p. 68.

(6) Ibidem, p. 10.

(7) Ibidem.

(8) N. BOHR, Physique atomique et connaissance humaine, Paris, Ed. Gontier, 1961 , p. 67.

(9) Ibidem, p. 12-14

(10) Voir W. HEISENBERG, Physique et philosophie. La science moderne en révolution, Paris, Albin Michel, 1961, p. 208.

(11) Voir BoHr, Atomic Theory and the Description of Nature, p. 96-100 et 117-119; Physique atomique et connaissance humaine, p. 147-151. 
biologie, par exemple, un aspect peut être envisagé comme résultant d'un système complexe de molécules en interactions. C'est l'approche " mécaniste ». Mais, on peut appréhender la réalité étudiée comme produite par les éléments constituant un organisme et en interactions, cellules, tissus, organes, etc. On a affaire alors à une approche " organiciste ». Dans le premier cas la notion de finalité n'intervient pas, elle est nécessaire dans le second. De la même manière un fonctionnement du psychisme peut être interprété comme la réponse aux stimuli de l'environnement ou comme le produit d'une structure capable de se développer et de s'adapter. Dans la même ligne, l'acte humain peut être perçu comme dépendant des contraintes internes et externes du psychisme ou comme le résultat de l'exercice d'un libre arbitre. Dans chacun des cas évoqués interviennent deux types d'observation, avec leurs conceptualisations, qui s'excluent mutuellement.

Éclairons ce point par l'exemple de la biologie tel que Bohr luimême l'a traité (12). La physique quantique a créé des conditions nouvelles pour l'étude de questions biologiques du point de vue de la physique. Celle-ci apporte la compréhension de phénomènes comme la photosynthèse ou des propriétés de composés compliqués telles la chlorophylle ou l'hémoglobine. Mais, ce que fournit la physique quantique ne suffit pas pour découvrir une explication d'ensemble des phénomènes biologiques (13). La biologie part toujours de l'hypothèse de la vie, le fait que l'organisme est vivant. Or, la science est fondée sur le postulat que dans la nature tout est soumis aux mêmes lois. En poussant l'analyse des processus biologiques aussi loin que celle des phénomènes atomiques, on n'obtiendrait pas pour les organismes un comportement différent de celui des corps inorganiques. Mais, les conditions de la recherche en biologie diffère de celles de la physique, car il s'agit de maintenir en vie ce qui est étudié. Cette limite empêche de connaître rigoureusement les processus physiques à l'œuvre. D'où, l'existence de la vie doit être considérée comme un fait élémentaire qui ne peut être fondé sur aucun autre et doit être pris comme point de départ de la biologie, de même que l'existence du quantum d'action, élément irrationnel du point du vue de la mécanique classique, forme, avec celle des particules élémentaires, la base de la physique atomique (14). Le développement de la compréhension des structures et des processus chimiques a ouvert des possibilités d'application de ces résultats en biologie. Cela n'implique pas pour autant de ne plus utiliser des arguments de type finaliste. La réponse à cette difficulté tient dans la prise en compte des conditions d'observation dans la définition des

(12) Voir BoHR, Physique atomique et connaissance humaine, p. 16-22; 34-39 et 161-165.

(13) Ibidem, p. 18.

(14) Ibidem, p. 20. 
concepts physiques. Les perspectives "mécanistes » et "finalistes " utilisées en biologie sont complémentaires parce qu'elles relèvent de deux types d'observation qui s'excluent mutuellement tout en étant indispensables pour décrire le plus complètement la vie (15). Jusqu'à présent, remarque Bohr, la définition des concepts biologiques n'avait pas pris en considération l'observateur et les conditions d'observation. C'est là ce qu'apporte la complémentarité. Elle exprime la relation qui existe entre des phénomènes observés dans des conditions expérimentales différentes. La complémentarité apporte un éclairage nouveau sur les deux manières d'approcher le vivant. Elle joue au plan épistémologique de ce qu'on peut connaître et pas au niveau ontologique de ce qui est.

Bohr a également posé les bases de l'application de la complémentarité à l'étude des religions (16). Il rappelle que la complémentarité en physique établit une relation entre les observations effectuées avec différentes conditions expérimentales choisies par l'observateur. Dans cette perspective, la complémentarité permet de rendre compte, selon Bohr, de la diversité des cultures et des traditions religieuses. Fondamentalement, en effet, celles-ci constituent des manières de vivre tant individuelles que collectives, théorisées dans des réseaux de connaissances et de croyances. Ces manières de vivre correspondent alors aux différentes conditions expérimentales rencontrées en science. La complémentarité montre que connaître l'être humain indépendamment de sa culture et de sa tradition religieuse est impossible, et que la connaissance qu'on peut en avoir est liée aux conditions d'observation, c'est-à-dire dans ce cas au mode de vie. Soulignons encore une fois que la notion de complémentarité intervient au plan de la connaissance, remarque importante sur laquelle nous reviendrons plus loin. Voyons auparavant comment des théologiens ont appliqué l'idée de complémentarité dans leur discipline.

Rappelons les principaux traits de la complémentarité (17). Une réalité nouvellement observée se révèle inexprimable totalement et de manière convaincante à l'aide des conceptions connues, mais suppose la mise en cuvre de deux conceptions contradictoires. Ces deux conceptions appartiennent à la même discipline et se rapportent à la même réalité tout en étant incompatibles entre elles.

Dans ces conditions, la complémentarité ne peut être appliquée telle quelle à la relation entre deux disciplines différentes, par cxcmple, pour

(15) Ibidem, p. 163.

(16) Voir N. BOHR, "Physical Science and the Study of Religions ", in coll., Studia Orientalia Ioanni Pedersen, Hauniae, 1953, p. 385-390.

(17) Voir J.E. Loder, W.J. Neidhart, « Barth, Bohr, and Dialectic », in W. Mark Richardson, Wesley J. Wildman (ed.), Religion and Science, p. 282-288. 
ce qui nous intéresse ici, entre science et théologie. Car, il s'agit alors de conceptualisations ne relevant pas de la même discipline. En revanche, la complémentarité peut être appliquée en théologie, là où deux jeux de conceptualisations s'avèrent nécessaires pour expliciter la foi chrétienne. La situation paradoxale de la théologie chrétienne rappelée au début n'invite-t-elle pas le théologien à employer à son tour le concept de complémentarité ?

En fait, les théologiens qui s'y sont essayés ont surtout appliqué la complémentarité en christologie (18).

La confession du Christ comme vrai Dieu et vrai homme place le croyant dans une situation qui rappelle celle de la physique quantique. En effet, nous avons bien affaire à une réalité nouvelle, le Christ Jésus, qui requiert pour l'approcher deux compréhensions traditionnelles séparées, celle de la divinité et celle de l'humanité. La première rend compte de l'existence du Christ Jésus, la seconde l'insère dans l'espace et le temps, c'est-à-dire dans le créé. De plus, il y a une asymétrie entre les deux conceptualisations : la divinité du Fils préexiste à son humanité, elle la rend possible et n'a pas de commune mesure avec elle. La complémentarité semble fonctionner à merveille, mais une observation attentive met en évidence les difficultés à résoudre.

En effet, la réflexion des Pères de l'Église et les conciles qu'elle a suscités apportent des conditions précises quant à l'utilisation de la notion de complémentarité. Cet enseignement donne comme condition qu'il y a dans le Christ Jésus simultanéité des deux natures sans séparation ni confusion. Les langages sont conjoints. Ils ne peuvent être confondus, ni séparés. Il y a en plus raccordement entre langage et ontologie. Les conciles affirment clairement l'objectivité du langage théologique et le réalisme qui l'accompagne : ce qui est dit correspond à ce qui est. Or, la notion de complémentarité implique de ne pas employer simultanément les langages portant sur les aspects complémentaires, c'est-à-dire ici le langage sur le divin et celui sur l'humain et ne se prononce pas, on l'a vu, quant à l'ontologie. Faire intervenir la complémentarité oblige à s'interroger sur le type d'objectivité du discours, c'est-à-dire sur le rapport entre le langage et la réalité.

Mais, la complémentarité peut encore apporter autre chose. Une autre voie à explorer consiste à prendre le principe de complémentarité avec le statut heuristique qu'il possède maintenant dans la présentation de la physique quantique. C'est la voie retenue par A. McGrath, de

(18) Pour une première approche des théologiens qui ont utilisé la complémentarité en théologie, voir A. Mc Grath, The Foundations of dialogue in science and religion, Oxford, Blackwell Publishers Ind, 1998, p. 200 sq. ; C. B. KaISER, "Quantum complementarity and Christological dialectic", in W. M. RICHARDSON, N. J. WILDMAN (ed.), Religion and Science, p. 294 sq. 
formation d'abord scientifique avant de devenir théologien (19). Dans cette perspective, la complémentarité rend compte de la formulation progressive du dogme christologique et exprime l'impossibilité de réduire le discours sur Jésus à un langage cohérent préexistant, que ce soit celui de l'humain ou celui du divin. Les Écritures et leur interprétation, la pratique de l'Église (liturgie, piété, etc.) ont contraint à juxtaposer les deux approches, humaine et divine, de Jésus selon des règles résultant de débats souvent difficiles et laborieusement définies par les conciles de Nicée 1 (325) à Constantinople 3 (680-681). Qu'apporte alors le principe de complémentarité à la théologie? Une méthode, c'est-à-dire comment sous la pression de situations nouvelles, le théologien voyant qu'il n'est pas possible de maintenir tel quel le langage antérieur, portant sur la divinité ou sur l'humain en relation avec le divin, peut entrer dans la construction d'un nouveau langage théologique en utilisant des modèles qui sont dérivés de situations connues et comprises (20). Pour la théologie chrétienne, ces modèles proviennent de la révélation et de son interprétation. Il s'agit comme en physique de construire une nouvelle théorisation à partir de termes et de concepts connus, qui de ce fait voient leur contenu renouvelé. Il s'agit alors d'envisager le rapport entre connaissance et réalité.

Nous avons vu comment la notion de complémentarité a été appliquée en théologie soit à l'élaboration du contenu d'une doctrine donnée, soit à la dynamique ayant abouti à la constitution de cette doctrine. On attendait de cette notion qu'elle ouvre la voie à une nouvelle manière d'articuler les propositions contradictoires caractérisant la théologie chrétienne. Ce n'est pas le cas. Mais, à regarder de plus près cette notion telle que Bohr l'a définie, on voit qu'elle concerne le problème fondamental du rapport entre langage, connaissance et réalité. $\mathrm{La}$ complémentarité contribue à situer l'objectivité du discours en obligeant à se poser la question de son rapport au réel. À ce point de vue, cela vaut la peine de persévérer.

\section{Langage, connaissance et réalité}

Pour Bohr, la complémentarité introduit un changement fondamental dans la manière dont l'être humain connaît les choses. Analysant la manière dont les physiciens des premières décennies du $20^{\mathrm{e}}$ siècle ont procédé pour rendre compte des processus physiques à l'œuvre dans la matière et ses constituants, Bohr met en évidence deux faits importants.

(19) Voir MCGRath, p. 201-205.

(20) Ibidem, p. 205 
Le premier est qu'on part de ce qui est connu pour appréhender ce qui ne l'est pas en transposant du premier domaine au second concepts, instruments et méthodes. C'est ce qu'on appelle le principe de correspondance (21). Ainsi, des notions comme celles de particule ou d'onde, des grandeurs comme celles de position ou de vitesse, sont reprises de la physique classique pour être appliquées dans le nouveau domaine qu'est la physique de l'atome.

Le second fait porte, on l'a vu, sur l'impossibilité de séparer, à la différence de ce qui se passe en physique classique, l'instrument de mesure du système quantique observé. A cause de la disproportion qui existe entre les deux, on ne peut plus négliger l'interaction entre les deux.

Il y a en fait une double disproportion. L'une qui est matérielle est celle qui existe entre le système observé microscopique et l'instrument d'observation macroscopique. L'autre est conceptuelle. La physique quantique part de concepts construits à l'aide de l'expérience du monde macroscopique pour les appliquer au domaine microscopique. Ainsi, l'irréductibilité du lien entre instrument de mesure et système physique ajoutée à l'obligation d'utiliser des concepts et des méthodes issus du domaine macroscopique fait qu'il faut définir leurs conditions d'utilisation dans ce nouveau domaine (22). Les observations ne fournissent pas des données sur le système quantique observé, mais sur l'ensemble constitué par le système et l'instrumentation qui opère la mesure et dépend de l'observateur, comme le souligne Bohr :

Il est également important de comprendre que, de ce fait, aucun renseignement sur une phénomène qui se trouve, en principe, hors du champ de la physique classique, ne peut être interprété comme une information sur des propriétés indépendantes des objets : ce renseignement est intrinsèquement lié à une situation définie, dont la description implique essentiellement les appareils de mesure en interaction avec les objets (23).

Si la complémentarité consiste d'abord à prendre en compte dans la nouvelle physique la non séparabilité entre le système et l'instrument de mesure, elle oblige également, selon Bohr, à repenser le rapport entre langage, connaissance et réalité.

La description des phénomènes naturels fait intervenir des résultats d'observation. Or, pour rendre compte d'une expérience, qui met en œuvre le système et l'instrument de mesure, on ne peut que se servir

(21) Voir Messiah, vol. 1, p. 24-25 et 51-60. Ce principe signifie qu'à la limite où le quantum est nul on retrouve les lois de la physique classique; ibidem, p. 25 et 180.

(22) Voir N. BoHr, Atomic Theory and the Description of Nature, p. 53.

(23) BOHR, Physique atomique et connaissance humaine, p. 44. 
de la terminologie de la physique classique. Mais, à l'échelle microscopique, on ne peut plus séparer l'instrument du système observé. Il en résulte que les résultats d'observations effectuées dans des conditions expérimentales différentes, et donc selon des conceptualisations différentes, ne peuvent être rassemblés en une image unique, mais regardés comme complémentaires au sens où chaque résultat épuise les informations accessibles concernant le système étudié dans les conditions expérimentales indiquées (24). Les résultats se complètent, mais ne peuvent être présentés de manière cohérente en un seul tableau. Les concepts ne peuvent plus être utilisés tous en même temps. Le sens des concepts et donc du langage employé dépend des conditions expérimentales. La complémentarité est donc une notion scientifique, mais elle possède aussi des implications sur ce que sont la connaissance, la science et le réel :

Pour désigner la relation qui existe entre les phénomènes observés sous les conditions expérimentales différentes, on a introduit le terme de complémentarité, afin de rappeler que c'est seulement par leur ensemble que de tels phénomènes épuisent la totalité des renseignements définis que l'on peut avoir sur les objets atomiques. [...] le point de vue de la complémentarité résulte immédiatement de notre situation d'observateurs dans un domaine de l'expérience où l'application univoque des concepts servant à la description des phénomènes dépend essentiellement des circonstances de l'observation (25).

Si l'on veut connaître la position d'une particule de manière précise, alors la valeur de sa vitesse ne pourra l'être, et inversement. On dit que ces variables sont complémentaires. La description par le moyen du langage de la physique classique des propriétés des systèmes quantiques nécessite la répartition des grandeurs de la physique classique par paires de grandeurs complémentaires. L'une des grandeurs relève du formalisme « corpuscule » et l'autre du formalisme " onde ». L'augmentation de la précision sur un membre de la paire s'accompagne d'une perte correspondante sur la précision de l'autre (26).

La dualité onde-corpuscule exprime à sa manière la complémentarité, c'est-à-dire le caractère complémentaire des analogies " corpuscule " ou " onde » (27). La notion de dualité est cependant ambiguë. S'agit-il d'une dualité de formalismes et donc de langages, ou bien d'une dualité au sein de la réalité elle-même? A ce point de vue, la complémentarité va plus loin au plan épistémologique, car elle exprime

(24) Voir Messiah, vol. 1, p. 129.

(25) BOHR, Physique atomique et connaissance humaine, p. 161

(26) Voir MESSIAH, vol. 1, p. 128-134.

(27) Voir BoHr, Physique atomique et connaissance humaine, p. 45. 
le caractère instrumentaliste de la physique quantique. En effet, toutes ses conclusions se mettent toujours sous la forme suivante : «On obtient tel résultat si l'on effectue telle ou telle observation » (28). Pour Bohr, il est clair que l'accent est mis sur le langage : la complémentarité est d'abord celle des formulations théoriques. La perspective de la complémentarité est avant tout descriptive et fonctionnelle, même si elle suppose la réalité de ce qui est décrit et étudié (29). Conséquence épistémologique, la physique quantique pose que la science étudie des phénomènes, pas des objets (30). De plus, le formalisme quantique ne décrit pas ce qui se passe dans le temps mais une tendance des phénomènes et de la connaissance qu'on en a (31). De ce fait, une certaine conception du réel est engagée. C'est pourquoi, la complémentarité a aussi une signification ontologique qui là aussi la distingue de la dualité.

Pour éclaircir ce point important, il faut considérer que les deux formalismes et les deux types d'expérience qu'ils permettent engagent deux compréhensions différents et exclusives du système étudié qui en physique classique vont ensemble. En effet, selon elle, suivre un phénomène dans le temps revient à suivre l'évolution dans l'espace et dans le temps des variables qui caractérisent le système. Cette évolution est déterminée par les lois physiques qui rendent compte de l'évolution d'un système en fonction de ses caractéristiques et des contraintes extérieures. Dit autrement, la description spatiotemporelle du système est en même temps une explication causale de son processus d'évolution. Ce n'est plus le cas en physique quantique. Du fait de l'existence d'un quantum d'action fini et indivisible, il y a séparation entre la description spatiotemporelle et l'explication causale, entre la mise en évidence d'un événement et celle de la cause d'un processus (32).

En effet, déterminer la position d'un objet quantique, c'est l'inscrire dans l'espace et le temps, c'est donc le manifester comme événement . C'est le cas par exemple de la radioactivité naturelle de certains atomes. Il s'agit d'observer la désintégration d'un atome : elle a lieu ou non. Le formalisme ne donne pas les raisons de la désintégration mais fournit les prévisions quant à la désintégration d'atomes ici et maintenant. L'autre formalisme, complémentaire, ne répond pas à ce genre de question, mais il met en évidence un phénomène en tant que résultat d'une action précise. Il rend compte par exemple de l'émission d'un rayonnement par un atome ayant reçu auparavant de l'énergie. La complémentarité « onde »-« corpuscule » entraîne celle qu'il y a entre

(28) MESSIAH, vol. 1, p. 127.

(29) Voir MacGrath, p. 196.

(30) Bohr se situe dans la lignc de la distinction kantienne entre la chose en soi et le phénomène, la chose manifeste à laquelle l'être humain a accès.

(31) Voir HEISENBERG, p. $36 \mathrm{~s}$.

(32) Voir BoHr, Atomic Theory and the Description of Nature, p. 54-55 et 93-94. 
la manifestation d'un événement et la cause d'un processus (33). On découvre par ces exemples que si les notions de dualité et de complémentarité sont proches l'une de l'autre, elles ne se recouvrent cependant pas totalement. Ce n'est pas pour rien que Bohr préfère parler de complémentarité. La différence se situe dans la portée ontologique et donc épistémologique, à savoir sur ce que peut connaître la science. La dualité introduit, au moins implicitement, un jugement sur ce qu'est un système quantique et sur la permanence de ses propriétés, tandis que la complémentarité porte sur les résultats d'expérience et donc sur la manière dont se manifeste un système quantique. On reste dans l'ordre des phénomènes sans chercher à se prononcer sur ce que serait la réalité en dehors des observations qu'il est possible de faire en physique.

C'est d'ailleurs cette réserve, pour ne pas dire cette limite, qu'ont toujours défendue Bohr et les tenants de l'école dite de Copenhague (34). C'est encore ce qu'illustre la discussion entre Bohr et Einstein lors des congrès Solvay de 1927 et 1930 et qui a conduit à la controverse associée au paradoxe soulevé par Einstein et ses collaborateurs Podolsky et Rosen, d'où le nom de paradoxe EPR, auquel Bohr a répondu (35). Le débat porte sur la notion d'élément de réalité correspondant à une grandeur physique d'un système donné et dont on peut prédire la valeur sans perturber le système et qui de ce fait existe indépendamment de toute observation. C'est la perspective défendue par Einstein. Elle oblige à postuler la séparation entre système et instrument de mesure. Or, ce faisant, rappelle Bohr, on attache des attributs physiques à des objets impliqués dans des phénomènes qui ne permettent aucune distinction nette entre le comportement propre de ces objets et leur interaction avec les instruments de mesure (36). L'interprétation correcte des phénomènes quantiques interdit par conséquent de séparer " objet » et instruments de mesure, ce qui conduit d'une part à distinguer deux types de langage selon les expériences et d'autre part à considérer non plus des objets mais des phénomènes. En physique quantique, la connaissance, et par conséquent le langage utilisé, ne porte plus, comme en physique classique, sur des objets, mais sur des phénomènes. Comme ceux-ci

(33) Voir MACKINNON, p. 265 ; C.B. Kaiser, op. cit., p. 293.

(34) Voir le chapitre intitulé "L'interprétation de Copenhague " in Heisenberg, p. $33-51$.

(35) Voir BoHR, Physique atomique et connaissance humaine, p. 77-100; la controverse figure dans les deux articles : A. Einstein, B. Podolsky, N. Rosen, "Can Quantum-Mechanical Description of Physical Reality Be Considered Complete?", Physical Review 47, 1935, p. 777-780 ; N. Bohr, "Can Quantum-Mechanical Description of Physical Reality Be Considered Complete? ", Physical Review 48, 1935, p. 696702 .

(36) Voir BoHr, Physique atomique et connaissance humaine, p. 99. 
dépendent des conditions d'observation, et donc des choix de l'observateur, l'objectivité atteinte est faible. La physique classique suppose au contraire une objectivité forte selon laquelle il existe une correspondance entre le langage et des éléments constitutifs de la réalité étudiée. La connaissance acquise par l'expérience est indépendante du physicien. Elle l'est aussi de la méthode employée pour déterminer ces éléments, au sens où l'existence de ces éléments ne dépend pas de l'expérience. La physique quantique rompt avec cette tradition et considère une objectivité faible selon laquelle la connaissance acquise est indépendante de l'observateur mais pas de l'expérience effectuée qui détermine le phénomène produit comme interaction entre l'objet et l'instrumentation (37). En physique classique, on connaît des éléments de réalité, en physique quantique, on connaît des phénomènes. La complémentarité change le rapport entre la connaissance et ce qui est connu. En distinguant les langages, elle rend possible l'extension à la physique quantique du langage scientifique habituel et éclaire la compréhension des phénomènes à l'aide des représentations associées à ce langage. La complémentarité joue un rôle d'auxiliaire dans la connaissance des phénomènes. Mais, n'a-t-elle que cette fonction?

\section{Objectivité et complémentarité}

Soulignons pour commencer le rôle d'auxiliaire de la complémentarité en remarquant qu'elle n'intervient plus en physique qu'à un niveau heuristique. On ne cherche plus en effet à décrire les phénomènes quantiques dans un langage accessible. L'intérêt n'est plus par conséquent dans la découverte d'analogies avec les objets de la physique classique. Le formalisme mathématique de la physique quantique permet de formuler toutes les situations rencontrées et donc de retrouver les deux langages, " onde » et " corpuscule » (38). C'est un avantage incontestable de rigueur et de fonctionnalité. Ce formalisme en revanche a pour inconvénient son caractère abstrait si bien qu'on ne sait plus très bien ce que les énoncés et les résultats veulent dire d'un point de vue physique (39). On retrouve le dilemme du début de la physique nouvelle. Ou on essaie de s'approcher des intuitions classiques et on ne peut éviter l'imprécision, voire le flou, ou on utilise le formalisme et on est dans l'abstraction. Or, c'est précisément les deux pôles que

(37) B. D'Espagnat, Traité de physique et de philosophie, Paris, Fayard, 2002, p. 467-470, ainsi que ses autres ouvrages sur la question, en particulier, $A$ la recherche du réel. Le regard d'un physicien, Paris, Gauthier-Villars, $1981^{2}$ et Une incertaine réalité. Le monde quantique, la connaissance et la durée, Paris Gauthier-Villars, 1985.

(38) Voir HEISENBERG, p. 40-41.

(39) Voir R. Feynman, R. Leighton, M. SAnds, Le cours de physique de Feynman, 3, Mécanique quantique, Paris, InterEditions, 1979, p. 32. 
Bohr veut tenir en introduisant le concept de complémentarité : raccrocher rigoureusement des descriptions issues de la physique classique à une compréhension nouvelle et rigoureuse des phénomène atomiques. Il s'agit de raccorder le langage et la conception du monde quantiques au monde macroscopique, celui de l'expérience humaine ordinaire (40). Le souci de rechercher des analogies accessibles au domaine d'expérience habituel de l'être humain est d'ailleurs le trait qui sépare Bohr des physiciens qui partagent les mêmes conceptions physiques que lui, tel Heisenberg. Ce constat explique que la théorisation contemporaine de la physique quantique a honoré à sa manière les exigences posées par Bohr, mais sans faire appel à la complémentarité. Celle-ci est plutôt déduite du formalisme (41).

Cependant, la situation n'est pas tout à fait la même en physique et dans des disciplines comme la biologie, la psychologie ou l'éthique où Bohr a proposé d'étendre la notion de complémentarité. Car, pour ces dernières, les deux conceptualisations utilisées ressortissent au domaine d'expérience ordinaire de l'être humain. Il ne s'agit pas, à l'instar de la physique quantique, de modéliser un domaine inaccessible à l'expérience macroscopique ordinaire à l'aide d'outils théoriques élaborés dans ce dernier champ. Ce qui pousse à utiliser la complémentarité, c'est de ne pas arriver à rendre compte de la totalité phénoménale de la réalité étudiée à l'aide d'une seule théorie, mais qu'il faille recourir à deux points de vue incompatibles l'un avec l'autre. A quelles conditions peut-on alors appliquer le concept de complémentarité dans une telle situation? L'application suppose que la même réalité puisse être envisagée selon deux perspectives théoriques exclusives, c'est-à-dire qu'étant précisées les conditions expérimentales une seule s'applique. Les conditions seront que ces deux approches théoriques doivent premièrement se rapporter à la même réalité et être dans des langages de même type et deuxièmement qu'elles doivent avoir été validées dans le domaine envisagé (42). Autrement dit la pertinence de chacune est supposée déjà prouvée. Notons encore que la relation entre les deux conceptualisations n'est pas symétrique. Il s'agit d'un côté de constater l'existence, c'est-à-dire d'inscrire dans l'espace et le temps, de l'autre d'expliquer, c'est-à-dire d'inscrire dans un ordre causal. Dernier point, cette disjonction entre deux conceptualisations est liée à la prise en compte de l'être humain comme observateur de la réalité.

C'est ce qu'on peut remarquer en reprenant l'exemple de la biologie. Les formulations "mécaniste " et " organiciste » constituent bien

(40) A. MCGRATH, op. cit., p. 194-195.

(41) Sur cette question, voir R. OMNĖs, Comprendre la mécanique quantique, Les Ulis, EDP Sciences, 2000, en particulier p. 159-168.

(42) McGrath, p. 191-194 et I. Barbour, Religion and Science. Historical and Contemporary Issues, New York, Harper Collins, 1997, p. 170. 
deux conceptualisations qui ne sont pas symétriques. L'approche "mécaniste " cherche à expliquer structures et processus en jeu dans les organismes vivants, tandis que la formulation « organisciste " met en évidence l'existence des formes et des processus. D'un autre côté, on retrouve le fait que ces conceptualisations résultent de la situation de l'être humain comme observateur du vivant.

On remarque d'ailleurs que le fondement de la différence entre les deux conceptions du vivant illustrées ici tient dans le rapport de l'observateur à la réalité observée. Dans un cas, il considère l'organisme dans sa totalité, et par conséquent dans sa complexité, dans l'autre, il analyse une partie de l'organisme ou un des processus à l'œuvre en lui.

Cependant, le recours à la complémentarité en biologie n'est pas dû, comme en physique, à la non séparabilité entre instrument et objet. Il est dû à la complexité des organismes qui conduit à poser la vie comme donné fondamental doté d'une téléologie qui permet de penser structures et processus de l'organisme comme un tout organisé. Bohr se situe dans la ligne de Kant pour lequel la biologie diffère de la physique en ce que l'esprit humain requiert d'intégrer les explications partielles, procurées par l'application de la physique en biologie, dans une perspective finaliste (43). Bohr a progressivement élucidé le fait que la limitation des explications causales en biologie par les conditions expérimentales est plus qu'une impossibilité pratique, due à l'obligation de maintenir l'organisme en vie. Elle est d'ordre noétique et tient au fait qu'on affirme la nécessité de poser en premier l'existence de la vie comme phénomène fondamental. Bohr voit dans ce constat un parallèle entre la biologie et la physique. L'introduction d'arguments téléologiques en biologie va de pair avec celle des quantas en physique atomique (44). La complémentarité intervient en biologie pour des raisons noétiques. Cependant, l'analyse de son application en biologie montre que la complémentarité comporte aussi des implications ontologiques. En effet, l'organisme existe indépendamment de toute observation. Les deux langages, "mécaniste " et " organiciste ", ont pour objet de décrire la réalité biologique.

Cette remarque laisserait entendre que l'extension de la complémentarité à d'autres domaines que la physique dépendrait de la portée ontologique du concept de complémentarité. S'il en est ainsi, cela modifierait substantiellement la conception de la complémentarité, telle que Bohr l'a définie. Dans la perspective de Bohr, en effet, la notion

(43) Voir N. Roll - Hansen, "The application of complementarity to biology : From Nicls Bohr to Max Delbrück ", Historical Studies in the Physical and Biological Sciences 30, 2000, p. 419-420.

(44) Voir Bohr, Physique atomique et connaissance humaine, p. 22 ; Roll - Hansen, p. 421 s. et 438 . 
centrale est celle de phénomène. La complémentarité permet la description et la prédiction de phénomènes, c'est-à-dire du comportement d'une réalité donnée. Mais, avec cette approche, on ne se prononce pas sur cette réalité en elle-même. La perspective philosophique est descriptive et fonctionnelle et pas ontologique, même si elle suppose la réalité de ce qui est décrit et étudié (45). En effet, un phénomène, selon Bohr, résulte de la réunion de la réalité, de l'instrument d'observation et de leur interaction.

Parce que le non engagement ontologique de la complémentarité au sens de Bohr limite son application à d'autres domaines comme la théologie, une idée serait de repenser son rapport au réel. Cette perspective devrait permettre de mieux préciser le sens de l'objectivité du discours par rapport au sujet qui le produit et par rapport à l'objet de l'expérience.

\section{La complémentarité inscrite dans le réel}

L'idée d'élargir la notion de complémentarité en lui associant d'emblée une portée ontologique est à la base de la démarche suivie par T. Magnin dans sa recherche théologique où le concept de complémentarité occupe une place centrale (46).

Selon Magnin, la notion de complémentarité ouvre des perspectives, déjà remarquées par Bohr, mais qu'il n'a pu vraiment exploitées, faute d'une formulation plus engagée vis-à-vis de l'ontologie. Pour remédier à ce déficit, des idées nouvelles sont introduites, celles de tiers-inclus et de niveau de réalité, reprises respectivement à $\mathrm{S}$. Lupasco et B. Nicolescu (47).

L'idée de Lupasco tient dans l'observation que dans le réel deux aspects contradictoires peuvent coexister mais sous des formes différentes dont l'une est actuelle et l'autre potentielle (48). C'est le cas de l'énergie. Tout objet quantique possède une énergie qu'il manifeste selon les cas sous forme ondulatoire ou sous forme corpusculaire. Quand un objet se manifeste sous forme ondulatoire, cela signifie donc que son énergie s'est actualisée sous cette forme et par conséquent, selon Lupasco, que l'énergie sous son autre forme, cor-

(45) Voir MCGrath, p. 192.

(46) T. MAGNIN, Entre science et religion. Quête de sens dans le monde présent, Monaco, Ed. du Rocher, 1998. Ce livre est le résumé d'une thèse de doctorat : $\mathrm{T}$. Magnin, L'importance de la philosophie morale dans le dialogue entre science et théologie. Illustration à partir de l'analyse des fondements de l'idée de complémentarité en science, Lille, 1997 ( $=$ Thc̀sc).

(47) MaGnin, Thèse, p. 186-191 ; Entre science et religion, p. 59-66

(48) Magnin, Thèse, p. $188 \mathrm{~s}$. 
pusculaire, est devenue potentielle (49). La notion de tiers-inclus exprime que l'énergie existe en permanence sous les deux formes, ondulatoire et corpusculaire, l'une étant plus actualisée que l'autre, qui est davantage à l'état potentiel que la première. Appliqué à la physique quantique, le tiers-inclus qualifie l'état quantique comme état d'équilibre dynamique entre les aspects complémentaires, ondulatoire et corpusculaire, qui demeurent indissociables et relèvent d'une logique inclusive (et l'un et l'autre) et non d'une logique exclusive (ou l'un ou l'autre). Inscrire la contradiction dans la réalité elle-même, au lieu de la laisser au plan des phénomènes et du formalisme, rend mal à l'aise.

La notion de niveau de réalité (50) va précisément servir à distinguer la réalité et ses manifestations que sont les phénomènes (51). Cette notion met en jeu des caractéristiques ontologiques avec les conséquences épistémologiques qui les accompagnent. Un niveau de réalité est caractérisé en effet par l'ensemble des systèmes qui correspondent à ces caractères. La notion de niveau de réalité diffère ainsi de celle de niveaux d'organisation pour lesquels les propriétés ontologiques et épistémologiques restent les mêmes quand on passe d'un niveau à un autre. Ce n'est pas le cas en revanche quand on change de niveau de réalité. Ainsi, on peut distinguer le niveau de réalité macroscopique, atteint par la physique classique, où la causalité est locale et où il y a séparabilité des objets, du niveau de réalité quantique, en général, mais pas toujours, microscopique où jouent une causalité globale et la non séparabilité.

Magnin combine les approches de Lupasco et de Nicolescu. L'état quantique (le tiers-inclus) se situe à un autre niveau de réalité que celui des manifestations ondulatoires ou corpusculaires. A l'observation au plan phénoménal d'aspects contradictoires pour un même système correspond au niveau de l'état quantique des propriétés qui ne sont plus contradictoires puisque opère à ce niveau une logique inclusive. La logique du tiers inclus vaut en effet pour l'état quantique qui correspond à un autre niveau de réalité que celui des phénomènes observés. Cette approche rappelle l'analyse aristotélicienne des propriétés contradictoires attribuées à un même objet : la contradiction se situe au niveau logique de l'affirmation des attributs et non au niveau ontologique du substrat de ces propriétés qu'est l'objet. Car, ce ne n'est pas sous le même rapport que les deux attributs sont donnés en même temps à

(49) Remarquons au passage que les relations d'incertitude de Heisenberg interdisent les états purs où toute l'énergie serait actualisée sous une seule des deux formes. Il reste toujours une partie d'énergie actualisée sous l'autre forme.

(50) MaGnin, Thèse, p. $188 \mathrm{~s}$. et 230-231.

(51) Cela rappelle l'ontologie stoïcienne. 
l'objet (52). Cependant, alors que le substrat et ses propriétés constituent le même niveau de réalité chez Aristote, ce n'est plus le cas dans la perspective développée par Magnin, constituant par là son originalité.

Les deux conceptualisations complémentaires issues de la physique classique sont médiatisées et unifiées à un autre niveau de réalité, le niveau quantique. Les contradictions relèvent d'un niveau où vaut la logique exclusive du ou bien... ou bien. En revanche, l'état quantique se situe à un autre niveau, celui du tiers unifiant, où opère la logique inclusive du et... et (53). Le niveau quantique reprend les deux formulations selon des règles définies par la physique quantique, ce qui correspond à une rupture. On le voit l'élargissement de la notion de complémentarité par celles de tiers-inclus et de niveaux de réalité permet de passer d'une description en termes d'exclusive (ou... ou) à une description où prévaut l'inclusion (et... et). Cependant, il ne s'agit pas seulement d'une description phénoménologique mais aussi ontologique. La complémentarité selon Magnin dépasse ce que dit la physique quantique pour laquelle un phénomène n'est jamais considéré comme étant à la fois corpuscule et onde.

Cette manière de voir entraîne l'existence de deux types de complémentarité, selon que les approches complémentaires se situent au même niveau de réalité ou à des niveaux différents (54). La complémentarité, envisagée à des niveaux de réalité différents, ouvre des perspectives intéressantes. Il suffit d'en mentionner quelques-unes. En premier, ce type de complémentarité, par la distinction des niveaux de réalité, permet de poser la distinction entre sujet pensant et objet pensé, entre le vécu de l'intelligence et la compréhension qu'elle produit. Ensuite, la complémentarité ainsi redéfinie introduit la dimension temporelle dans l'approche du réel aux plans ontologique et noétique. Au plan ontologique, potentialisation et actualisation permettent d'intégrer les propriétés contradictoires manifestées par l'expérience. Au plan noétique, la complémentarité permet de poser l'unité des contradictoires et leur permanence dans la réalité, par exemple ordre et désordre, etc. Elle constitue enfin un outil de lecture de la complexité (55).

Mais, ce qui retiendra le plus notre attention, c'est que la complémentarité ainsi revue implique le réalisme et une objectivité forte. Les propriétés dont on parle à propos des phénomènes expriment quelque chose de la réalité. Ce qu'il y a lieu de préciser maintenant.

(52) MAGnin, Thèse, p. 212-213.

(53) MAGNIN, Entre science et religion, p. 97-98 et 103-105.

(54) MAGNiN, Thèse, p. 215.

(55) Sur tous ces aspects, voir par exemple, Thèse, p. 221-225. 


\section{Complémentarité, objectivité et réalisme}

La réalité elle-même est appréhensible et connue seulement par les phénomènes observés. Ces observations mettent en jeu formulations théoriques (concepts, lois, théories) et instruments. En physique classique, pour la plupart des scientifiques, chaque propriété définie par le formalisme et observée à l'aide de l'instrumentation correspond à un élément de la réalité étudiée, indépendamment de l'observation, perspective défendue par Einstein dans sa critique de la physique quantique. La physique quantique, selon l'interprétation de Bohr et de l'école de Copenhague, ne parle que des phénomènes et pas de la réalité en tant que telle. Même si celle-ci est en général supposée exister, elle demeure inaccessible, en tout cas directement. Le réel est voilé, pour reprendre l'expression de B. d'Espagnat (56). Avec la complémentarité redéfinie par Magnin, la réalité est considérée telle quelle selon une structure à deux niveaux. Le premier niveau, directement accessible aux scientifiques, est celui des phénomènes. A ce niveau apparaissent les phénomène qui relèvent de formalismes exprimant des propriétés contradictoires d'une réalité qui se situe à un autre niveau, celui de l'état quantique. De ce fait Magnin retrouve le réalisme : les propriétés connues correspondent à quelque chose du réel. Le discours retrouve une objectivité forte, car la connaissance porte sur des propriétés du réel qui existent indépendamment de toute observation.

Les positions ontologique et noétique de Magnin se distinguent aussi bien de celles que l'on rencontre habituellement en physique classique, et dont un bon représentant est Einstein, que de celles de Bohr et de l'école de Copenhague. En effet, les deux modes de description corpusculaire et ondulatoire constituent, on l'a vu, deux modes d'interprétation différents et exclusifs des phénomènes. Pour Bohr et l'école de Copenhague ce fait implique qu'on ne peut parler de la réalité en tant que telle puisque le discours dépend des conditions expérimentales. En clair, une propriété n'a d'existence que si et seulement si elle possède une signification, c'est-à-dire si et seulement si elle est mesurable, au moins théoriquement, dans les conditions expérimentales envisagées. Dit autrement, référence et signification sont inséparables. On accède au réel, par exemple la position d'un corpuscule (en prenant le langage classique), si celle-ci a un sens dans les conditions données, ce qui veut dire qu'elle est effectivement mesurable, c'est-à-dire que les conditions expérimentales imposent le formalisme corpuscule. Si on sait par avance que dans des conditions données la position n'est pas mesurable, cela entraîne que la notion de position $n$ 'a alors aucun sens et par voie de conséquence qu'on ne peut lui

(56) Voir n. 37. 
attribuer d'existence. L'école de Copenhague refuse toute réalité existant indépendamment d'une observation au moins possible. Tel est le sens profond de la complémentarité introduite par Bohr. La référence à une réalité n'a de signification que si elle est observable. Cette affirmation conduit à poser une objectivité faible. Parler d'objectivité faible signifie qu'elle résulte des conditions de vérification du discours scientifique. L'objectivité est faible parce qu'on ne peut attribuer d'existence à des éléments de réalité indépendamment des observations.

En revanche, Magnin redéfinit la complémentarité pour réintroduire le réalisme et une objectivité forte. On connaît la réalité, le niveau quantique, par l'autre niveau, celui des phénomènes, qui constituent des manifestations de cette réalité, mais se rapportant au même objet (57). La distinction entre les niveaux de réalité tient au changement de perspectives noétique et ontologique quand on passe d'un niveau à l'autre. Au niveau phénoménal joue la logique de l'exclusive tandis qu'au niveau de la réalité ultime opère la logique inclusive.

La complémentarité ainsi remodelée par Magnin entraîne cependant des différences selon les disciplines. Car, les niveaux de réalité ne sont pas les mêmes en science et en théologie. En effet, la portée ontologique des concepts en physique n'est pas directement ontologique. Onde et corpuscule constituent essentiellement deux modes d'interprétation des phénomènes qui tiennent à l'utilisation de concepts tirés de la physique classique dans un contexte nouveau. Ces concepts permettent d'interpréter les phénomènes constituant le premier niveau de réalité. Avec la complémentarité redéfinie par Magnin, on atteint le second niveau, celui de l'état quantique, par le biais de ses manifestations que sont les phénomènes. La portée ontologique au niveau des phénomènes tient dans la mise en évidence de propriétés ou dans la mesure de grandeurs qui relèvent des formalismes contradictoires de la théorie. Mais, au niveau quantique, la réalité est une, car les phénomènes sont considérés comme des manifestations de la même réalité. Les propriétés phénoménales ne sont d'ailleurs contradictoires qu'au niveau phénoménal. Au niveau de la réalité quantique, elles ne le sont plus, car elles ne sont pas considérées sous le même rapport, c'est-à-dire dans les mêmes conditions d'observation.

En théologie, la portée ontologique de la complémentarité est directe. Affirmer que Jésus est vrai homme et vrai Dieu signifie l'objectivité et la réalité de ces caractéristiques. En outre, les niveaux de réalité sont définis différemment en science et en théologie où la foi et donc la grâce sont supposées (58). Le premier niveau correspond à celui de l'expérience et de la connaissance ordinaires. À l'aide de l'intelligence

(57) Magnin, Thèse, p. $364 \mathrm{~s}$.

(58) Ibid., p. 366-370. 
seule, il s'agit de comprendre la révélation comme un donné. La révélation est alors considérée comme passive. Ce niveau de réalité est identique d'une manière avec celui des sciences. D'une autre manière non. Car, il s'agit de comprendre le monde et la vie, et c'est là que les apports des connaissances profanes jouent, en fonction de la révélation. A ce niveau apparaissent les données contradictoires. Le second niveau où se produit l'unification est celui de la foi. À ce niveau la révélation devient active, car l'intelligence est mue par Dieu.

Examinons comment la complémentarité redéfinie par Magnin opère au plan ontologique en théologie. Magnin applique la complémentarité en théologie, à l'aide de plusieurs exemples dont celui de la christologie. La complémentarité telle qu'il l'a élargie, lui donne de traiter de front et le contenu et le développement de la doctrine (59). La complémentarité est alors une démarche heuristique, elle guide la recherche dans sa compréhension du réel et dans la constitution d'un langage adapté à cet effet. Il s'agit de repérer d'abord les aspects contradictoires d'une réalité, ici le Christ Jésus tel que le Nouveau Testament le présente. Le premier niveau de réalité est celui du créé terrestre. Selon la révélation judéochrétienne en effet Dieu intervient dans le créé et ces interventions appartiennent au créé. Jésus est homme. En même temps il diffère des autres êtres humains par sa relation à Dieu. Il représente l'intervention dernière et suprême de Dieu en vue d'accomplir son dessein. Dans cette ligne, Jésus est présenté par la révélation comme à la fois humain et divin. Or, les propriétés de l'humain et du divin s'opposent en particulier dans la tradition culturelle gréco-latine. Nous avons donc bien deux modes de compréhension contradictoires concernant le Christ Jésus avec leurs langages propres, celui de l'humain et celui du divin.

Ces deux modes, et les langages qui leur sont associés, sont à utiliser pour parler de Jésus, puisqu'il est confessé comme étant une seule réalité. Il s'agit ensuite d'éprouver ces modes et de vérifier par la révélation et sa tradition interprétative qu'on n'a pas affaire à un phénomène déjà connu mais à un degré inobservé jusque-là. Tel n'est pas le cas. Aucun être humain n'est présenté dans la révélation de la manière dont l'est Jésus. Il n'existe pas non plus de moyen d'unifier ces modes de compréhension et leurs langages à ce niveau. On ne peut pas davantage réduire un des modes à l'autre. Par exemple, on pourrait dire que Jésus est en relation avec Dieu en tant que prophète, mais avec un charisme suréminent qui le distinguerait de tous les autres prophètes tout en faisant de lui un homme comme les autres. Dans une direction opposée, on pourrait penser qu'il est un être spirituel, une puissance angélique qui s'est manifestée sous forme humaine tout en

(59) Concernant la démarche, voir MAGNIN, Entre science et religion, p. 103-105. 
appartenant à la création céleste et qui se distinguerait des autres manifestations angéliques par sa durée, coextensive à une vie humaine, et par son intensité, en se présentant sous la forme d'une vie humaine et faisant de Jésus un semblant d'homme. La tradition chrétienne a rejeté ces deux voies, dans lesquelles on aura reconnu respectivement l'adoptianisme et le docétisme. Jésus est réellement Dieu et homme.

Puisque nous avons affaire à deux modes de compréhension contradictoires, il s'agit en une nouvelle étape de la réflexion de rechercher le principe d'unification à un autre niveau de réalité, celui de la foi. $\mathrm{Ce}$ fut le travail des conciles. A ce nouveau niveau de réalité, Jésus est en effet reconnu comme étant Dieu le Fils, différent du Père, mais Dieu au même titre que le Père. Sa divinité est identique à celle du Père et ne résulte pas d'une participation à la sienne qu'il aurait par grâce, ce qui reviendrait à dire qu'il est une créature. En même temps son humanité est bien comme la nôtre et donc Jésus n'est pas un être intermédiaire, Dieu animant lui-même un corps humain. Il n'est pas non plus Dieu habitant dans un homme, ce qui introduirait une dualité de sujets. Il possède réellement les deux natures, divine et humaine. Le principe d'unification est à chercher en Dieu lui-même. Dans ce sens les conciles affirment que Jésus est la personne du Fils qui a pris une nature humaine.

Mais, apparaît alors une nouvelle difficulté dans l'approche, car Jésus, le Père et l'Esprit sont réellement un tout en étant différents. Dieu se révèle comme Trinité. Le discours de foi concernant Dieu est lui-même marqué par une forme de contradiction qui tient aux langages descriptifs utilisés et qui présentent Dieu comme un et trine. La contradiction marque l'impossibilité de comprendre Dieu pour l'intelligence humaine créée, même en étant éclairée par la révélation. La contradiction est d'ordre noétique, pas ontologique (60). À son niveau de réalité, celui de la foi, Dieu ne peut être appréhendé tel quel par l'intelligence humaine aidée pourtant par son Créateur. Pour l'être humain, cette réalité se dédouble selon deux modes de compréhension rendus nécessaires pour rendre compte dans la foi du mystère de Dieu, deux niveaux de compréhension complémentaires concernant la divinité. Ils sont complémentaires mais toujours associés, et ne peuvent être utilisés l'un sans l'autre. Ils correspondent à la distinction de deux langages, celui de la personne et celui de la nature, qui sont par conséquent complémentaires mais doivent être employés ensemble. Mais, il ne s'agit pas d'introduire un nouveau niveau de réalité. On ne peut appliquer telle quelle la complémentarité redéfinie par Magnin dans l'approche du mystère de Dieu. Cela reviendrait à emprunter la voie du modalisme selon lequel les trois sont trois modes de manifestation de l'unique

(60) Ibid., p. 198. 
Dieu. En effet, la complémentarité consisterait à lever la contradiction entre les affirmations Dieu est un et Dieu est trine en considérant qu'elles correspondent à deux niveaux de réalité différents, celui de la manifestation de Dieu dans le créé et celui de Dieu en lui même, niveaux qui seraient respectivement identifiés au Dieu trine et au Dieu un.

Finalement, la notion de complémentarité retravaillée par Magnin réussit aux plans heuristique et noétique à montrer comment une recherche doit être guidée. Utilisée en théologie trinitaire, elle doit être limitée à l'action divine ad extra. Elle ne permet pas d'approcher le mystère de Dieu aussi bien que ne le fait la tradition théologique. Mais, derrière cette limite au plan ontologique, on retrouve le fait que la complémentarité, que Bohr avait mis en évidence, est un concept qui intervient au plan noétique et pas ontologique. La complémentarité conduit en effet à poser la question de la constitution d'un langage qui permette d'exprimer correctement ce que l'on arrive à connaître de la réalité expérimentée. La complémentarité est donc un guide pour construire un langage qui serve d'interface entre la pensée et le réel. Grâce à la complémentarité, on peut élaborer un langage abstrait et cohérent, scientifique ou théologique, avec lequel on peut parler correctement de ce qui est saisi dans l'expérience que l'on a du réel. Il s'agit de l'observation en science, de la vie de foi en théologie. Mais, le contenu de la pensée, concepts et images, n'a plus de référence empirique directe, comme dans la vie ordinaire.

L'intérêt de la complémentarité se situe bien au plan noétique et pas au niveau ontologique. La complémentarité concerne la relation entre sujet connaissant et objet connu, relation qui est au cœur du problème de la connaissance (61). Parce que la complémentarité change la compréhension de la relation entre sujet observant et objet observé, elle déplace l'articulation entre la volonté de chercher à saisir ce que l'on veut connaître et la possibilité de l'exprimer de manière cohérente. Elle montre que la frontière est fonction autant des conditions de l'observation, où le sujet est impliqué, que de l'objet observé et met fin à la distinction nette et fondamentale dans la science classique entre connaissance objective et croyance subjective (62). Il n'y a pas d'un côté les sciences objectives et de l'autre les croyances, philosophiques, religieuses ou autres.

La complémentarité intervient en montrant l'impossibilité de connaître entièrement le réel selon une modalité unique et donc avec

(61) Voir M. KATSUMORI, « Bohr's Early Complementarity Argument », Historia Scientarum, 8, 1998, p. 9.

(62) Voir BoHR, "Physical Science and the Study of Religions », p. 386; KATSUMORI, p. 16. 
un seul langage. Il en faut au moins deux qui correspondent à deux modes de manifestation de la réalité. Reste ouverte la question de la portée ontologique de chacun de ces modes, en science ou en théologie. La portée ontologique de ces modes détermine l'objectivité du discours correspondant. En physique quantique, les phénomènes se situent au même niveau ontologique. De ce fait les deux modes de manifestation engagent le même type d'objectivité faible. La situation diffère en théologie, car la manifestation du Christ Jésus relève d'une autre phénoménalité que celle des réalités créées. Or, l'objectivité atteinte est fonction de la manière dont se manifeste la réalité étudiée.

La notion de complémentarité se présente en définitive comme un intermédiaire dans la recherche, une aide qui permet de mettre en évidence l'existence de différentes modalités de connaissance correspondant chacune à une modalité phénoménale. Une fois cette découverte effectuée, la complémentarité peut céder la place à une reformulation du champ théorique selon les conditions d'observation des phénomènes. Une remarque le confirme. La physique quantique s'est constituée dans les dernières décennies selon $\mathrm{d}$ 'autres formalismes où les principes de complémentarité et de correspondance se déduisent des points de vue formels employés. La complémentarité est utilisée essentiellement pour présenter la physique quantique et introduire au formalisme (63).

Les considérations précédentes montrent que l'établissement de l'objectivité d'un discours demande d'examiner auparavant les modes de manifestation de la réalité envisagée. Dans cette perspective, parler du monde comme objet de science et en parler comme création relèvent de deux phénoménalités différentes. La phénoménalité du monde comme création est elle-même dépendante d'un autre mode de manifestation, celui de Jésus comme Fils de Dieu. On voit ainsi que l'objectivité du discours en science ou en théologie reste une question importante, mais qui renvoie à celle, plus fondamentale, des modes de manifestation du réel. 